\title{
Neoadjuvant intra-arterial chemotherapy combined with radiotherapy and surgery in patients with advanced maxillary sinus cancer
}

\author{
Won Taek Kim, MD¹, Jiho Nam, MD², Yong Kan Ki, MD¹, Ju Hye Lee, MD¹, Dong Hyun Kim, MD¹, \\ Dahl Park, PhD¹, Kyu Sup Cho, MD³ ${ }^{3}$ Hwan Jung Roh, MD ${ }^{4}$ Dong Won Kim, MD, PhD ${ }^{1}$ \\ 'Department of Radiation Oncology, Biomedical Research Institute, Pusan National University Hospital, Pusan National \\ University School of Medicine, Busan; ${ }^{2}$ Department of Radiation Oncology, Pusan National University Yangsan Hospital, \\ Yangsan; ${ }^{3}$ Department of Otolaryngology, Biomedical Research Institute, Pusan National University Hospital, \\ Pusan National University School of Medicine, Busan; ${ }^{4}$ Department of Otolaryngology, Pusan National University \\ Yangsan Hospital, Pusan National University School of Medicine, Yangsan, Korea
}

Purpose: The optimal treatment of advanced maxillary sinus cancer has been challenging for several decades. Intra-arterial chemotherapy (IAC) for head and neck cancer has been controversial. We have analyzed the long-term outcome of neoadjuvant IAC followed by radiation therapy (RT) and surgery.

Materials and Methods: Twenty-seven patients with advanced maxillary sinus cancer were treated between 1989 and 2002. Five-fluorouracil (5-FU, $500 \mathrm{mg} / \mathrm{m}^{2}$ ) was infused intra-arterially, and followed by RT (total $50.4 \mathrm{~Gy} / 28$ fractions). A planned surgery was performed 3 to 4 weeks after completion of IAC and RT.

Results: At a median follow-up of 77 months (range, 12 to 169 months), the 5-year rates of overall survival in all patients were $63 \%$. The 5 -year rates of overall survival of stage $\mathrm{T} 3 / \mathrm{T} 4$ patients were $70.0 \%$ and $58.8 \%$, respectively. Seven of fourteen patients with disease recurrence had a local recurrence alone. The 5 -year actuarial local control rates in patients with stage $T 3 / T 4$, and in all patients were $20.0 \%, 32.3 \%$, and $27.4 \%$, respectively. Overall response rate after the completion of IAC and RT was $70.3 \%$. During the follow-up, seven patients (25.9\%) showed mild to moderate late complications. The tumor extent (i.e., the involvement of either orbit and/or base of skull) appeared to be related with local recurrence.

Conclusion: Neoadjuvant IAC with 5-FU followed by RT and surgery may be effective to improve local tumor control in the patients with advanced maxillary sinus cancer. However, local failure was still the major cause of death. Further investigations are required to determine the optimal treatment schedule, radiotherapy techniques and chemotherapy regimens.

Keywords: Maxillary sinus neoplasms, Intra-arterial infusion, Radiation therapy, Surgery

\section{Introduction}

Malignant tumor of maxillary sinus is an uncommon neoplasm. It accounts for less than $0.5 \%$ of all cancers [1]. The majority of these tumors are diagnosed at locally advanced stage. The optimal treatment of locally advanced maxillary sinus cancer has been challenging for several decades. Extensive surgical resection including orbital exenteration was occasionally

Received 2 April 2013, Revised 1 June 2013, Accepted 21 June 2013.

Correspondence: Jiho Nam, MD, Department of Radiation Oncology, Pusan National University Yangsan Hospital, 20 Geumo-ro, Yangsan 626-787, Korea. Tel: +82-55-360-3453, Fax: +82-55-360-2449, E-mail: jihonam@pusan.ac.kr

(c) This is an Open Access article distributed under the terms of the Creative Commons Attribution Non-Commercial License (http://creativecommons.org/ licenses/by-nc/3.0/) which permits unrestricted non-commercial use, distribution, and reproduction in any medium, provided the original work is properly cited.

www.e-roj.org 
required to achieve complete resection of the tumor. However, these tumors are usually located in the vicinity of the critical organs, therefore, extensive surgery alone usually results in various complications and/or poor cosmetic outcomes. Radiation therapy (RT) alone, often performed in medically inoperable patients, was less effective than surgery alone or combined surgery and RT [2]. In spite of extensive surgical resection with or without $\mathrm{RT}$, the majority of treatment failure was local recurrence $[2,3]$.

Although the studies of combined modality approaches in patients with locally advanced maxillary sinus cancer have reported improved survival outcomes, however, combined chemotherapy may also increase the incidence of various adverse events. To minimize these adverse effects of chemotherapeutic agents, several studies of intra-arterial chemotherapy (IAC) combined-multimodal approaches have been performed in locally advanced maxillary cancer. However, the optimal treatment sequences and combinations still remain controversial.

Since 1989 we have treated patients with locally advanced maxillary sinus cancer using sequential multimodal therapy which consisted of neoadjuvant IAC, sequentially combined RT, and surgery. This study is an analysis of long-term followup data to evaluate the clinical outcomes of this combined modality approach in patients with non-metastatic locally advanced maxillary sinus cancer.

\section{Materials and Methods}

This retrospective study was performed by reviewing the patients' medical records and radiographic imaging data of the patients who had been diagnosed with locally advanced maxillary sinus cancer and then treated with multimodal therapy of IAC between November 1989 and December 2002.

\section{Patients}

Patients with locally advanced, non-metastatic cancer (cT34NOMO) of the maxillary sinus were analyzed for this study. All patients underwent pretreatment staging workup of a complete history and physical examination with clinical staging, complete blood cell count, liver function tests, renal function tests, chest X-ray, computed tomography (CT) scan, and ultrasonography of liver. Patients with the Eastern Cooperative Oncology Group (ECOG) performance status 0-2, no previous treatment, normal blood cell count, normal or less than 50\% increased level of liver and renal function test, and no other medical condition were combined. Written informed consents before treatment were provided by all of the patients.

\section{Intra-arterial chemotherapy}

Chemotherapy was administered intra-arterially. The intraarterial infusion was performed with the catheterization of superficial temporal artery. Through the skin incision in the preauricular region, the superficial temporal artery was exposed and the tip of the catheter was inserted into the internal maxillary artery. To identify the proper location of the feeding artery, Indigo carmine dye was infused, and then the catheter was advanced to the feeding artery. After the location was confirmed, the catheter was fixed.

Chemotherapeutic agent of 5-fluorouracil (5-FU, $500 \mathrm{mg} /$ $\mathrm{m}^{2}$ ) was infused at the rate of $1 \mathrm{mg} / \mathrm{m}^{2}$ per minute ( 8 hours per day) through the catheter. This was repeated every 4 to 6 days per week for 1 to 2 weeks. According to the tolerance and toxicity of the treatment, chemotherapy dose was reduced gradually by $25 \%$ to $50 \%$.

\section{Radiation therapy}

RT was started 1 to 2 weeks after the last course of the chemotherapy (IAC). All patients were treated with externalbeam RT using a 6-megavolt X-ray of linear accelerator. Either parallel opposed lateral fields or anteroposterior fields were used to treat the primary tumor area with a dose of 50.4 Gy at $1.8 \mathrm{~Gy} /$ fraction to the isocenter. Wedges or tissue compensators were used to maintain dose homogeneity within 10\% of the prescribed dose. RT was delivered with a continuous course of once-daily, 5 days-per-week treatment for all patients. The initial treatment volume was designed to encompass the primary tumor site with median $2 \mathrm{~cm}$ (range, 1 to $3 \mathrm{~cm}$ ) margins depending on CT or endoscopic findings and the proximity of critical structures. Elective neck irradiation to the ipsilateral neck was performed using anteroposteriorposteroanterior photon fields in patients with T4 stage, poor responders to chemotherapy (i.e., <PR) and good general condition. Total delivered dose to the ipsilateral neck was median 45 Gy (range, 42 to 50.4 Gy) at 1.8 Gy/fraction. Reirradiation was considered after surgery for patients with surgical margin involvement or suspicious residual disease.

\section{Evaluation of treatment response}

Response to the therapy was evaluated within 1 week after completion of two courses of IAC, before RT initiation, and 1 month later from RT completion. Using standard World 
Table 1. Patient characteristics $(n=27)$

\begin{tabular}{lc}
\hline \multicolumn{1}{c}{ Characteristic } & No. of patients \\
\hline Gender (male:female) & $25: 2$ \\
Age (yr), median (range) & $57(32-73)$ \\
T stage & \\
T3 & 16 \\
T4 & 11 \\
N stage & \\
N0 & 27 \\
Pathologic type & \\
Squamous cell carcinoma & 26 \\
$\quad$ Well differentiated & 8 \\
Moderately differentiated & 11 \\
Poorly differentiated & 7 \\
Adenocarcinoma & 1 \\
\hline
\end{tabular}

Health Organization criteria, tumor response was evaluated based on CT or magnetic resonance imaging (MRI) studies. Complete response (CR) defined as the state of disappearance of the gross lesions in the CT scan or MRI. Partial response (PR) was considered when at least a 50\% decrease in tumor size was apparent. Stable disease was defined as a less than 50\% decrease or a less than 25\% increase of measurable tumor size. Progressive disease was determined when there was an increase of at least 25\% tumor size or appearance of a new lesion.

Adverse side effects were recorded according to the National Cancer Institute Common Toxicity Criteria (CTC) ver. 2.0 for the chemotherapy and the Radiation Therapy Oncology Group for the RT.

The patients were followed closely by a medical oncologist, otolaryngologist, and radiation oncologist with physical examinations and CT scans or MRI of the head every 3 to 6 months.

\section{Surgery}

All patients underwent a planned surgical resection 3 to 5 weeks following IAC and RT. The extent and type of the surgery was decided according to the tumor response. Surgery consisted of a subtotal maxillectomy, a total maxillectomy, or a radical resection via a craniofacial approach. Orbital exenteration was considered when the apex or floor of the orbit was involved. Planned neck dissection was not performed electively.

\section{Statistics}

The endpoints of this study were the evaluation of the objec-
Table 2. Treatment characteristics

\begin{tabular}{lr}
\hline Characteristic & No. of patients \\
\hline Intra-arterial chemotherapy & \\
Total dose $\left(\mathrm{mg} / \mathrm{m}^{2}\right)$ & \\
$\geq 5,000$ & $18(67)$ \\
$<5,000$ & $9(33)$ \\
Radiation therapy & \\
Total dose (Gy) & $22(81)$ \\
$\geq 50.4$ & $5(19)$ \\
$<50.4$ & \\
Elective neck irradiation & $5(19)$ \\
Yes & $22(81)$ \\
No & \\
Surgery & \\
Type & $3(11)$ \\
Endoscopic resection & $7(26)$ \\
Partial maxillectomy & $13(48)$ \\
Total maxillectomy & $4(15)$ \\
Craniofacial resection &
\end{tabular}

tive tumor response and the toxicity and the survival rate. The survival rate was calculated from the date of the first treatment. The Kaplan-Meier method was used to estimate the rates of overall survival and actuarial local tumor control. The log-rank test was performed for the assessment of statistical significance.

\section{Results}

\section{Patients and treatments characteristics (Tables 1 and 2)}

Total thirty-two patients with previously untreated locally advanced, non-metastatic maxillary sinus cancer were treated with the combined neoadjuvant therapy and surgery between November 1989 and December 2002. Twenty-seven patients were available for the current analyses. Four patients were excluded because it was impossible to obtain sufficient information about the follow-up results from reviewing their medical records or calling the patients and different pathologic type. One patient had a synchronous primary malignancy in the lung. The median age of the patients was 57 years (range, 32 to 73 years). The majority of the patients were male (93\%, 25/27). Twenty-six patients had squamous cell carcinoma. All patients were clinically node negative at initial staging workup. Six patients initially presented with the orbital involvement.

\section{Patterns of failures and survival analysis}

1) Treatment responses: Overall response rate (complete 
Table 3. Patterns of recurrences

\begin{tabular}{ll}
\hline Characteristic & No. of patients \\
\hline Site of local recurrence & \\
Ethmoidal sinus & $5(19)$ \\
Nasal cavity & $2(7)$ \\
Cavernous sinus & $2(7)$ \\
Orbit & $3(11)$ \\
Subcutaneous tissue & $2(7)$ \\
Site of distant metastasis & \\
Lung & $2(7)$ \\
Bone & $1(4)$ \\
\hline
\end{tabular}

response plus partial response) of IAC was 55.5\% (T3-63\% vs. T4-45\%), and tumor down staging rate was 26\% (31\% vs. $18 \%$ ). With the completion of RT, the response rate was increased up to $70.3 \%$, and tumor down staging was $48.1 \%$. Three patients of clinical CR after RT showed pathologic $\mathrm{CR}$ with the surgery of endoscopic resection. Surgery was performed with several techniques according to the size and location of residual tumors. Total maxillectomy was performed in 13 patients (48\%). Seven patients (26\%) had a partial maxillectomy, and four patients (15.0\%) had a craniofacial resection. For one patient who had progressive disease with a positive neck lymph node after IAC, total maxillectomy and selective neck dissection were performed. Complete resection was possible for 19 patients (70\%). With a positive or close margin, the patients had received either an adjuvant RT (22\%) or a systemic chemotherapy (4\%).

2) Local and regional recurrence: During the follow-up period, there were total 14 recurrences observed (Table 3). Most local recurrences were occurred within 5 years after the treatment. Ten patients recurred at the orbit or the vicinity of skull base (i.e., ethmoidal sinus, cavernous sinus). Most common site of local recurrence was ethmoidal sinus. The actuarial 5-year rates of local recurrence in each stage group (stage T3 and T4) were 20.0\% and 32.3\% ( $p=0.568$ ) (Fig. 1). There was no regional recurrence in the subgroup of patients treated with the elective neck irradiation. However, two distant metastases were reported in the patients who were received the elective neck irradiation. Elective neck irradiation was performed in overall five patients. They were poor responders (i.e., <PR) to induction chemotherapy, T4 stage, and younger age with relatively good general condition.

3) Survival: At a median follow-up of 77 months (range, 12 to 169 months), the 5-year rates of overall survival in the patients

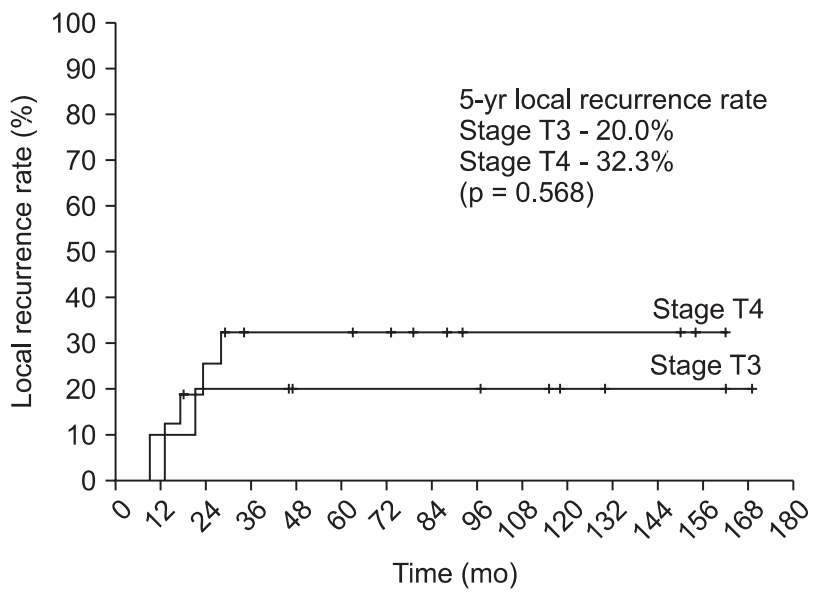

Fig. 1. Local recurrence rates according to the stage (T3 vs. T4) following combined multimodal therapy with neoadjuvant intraarterial chemotherapy.

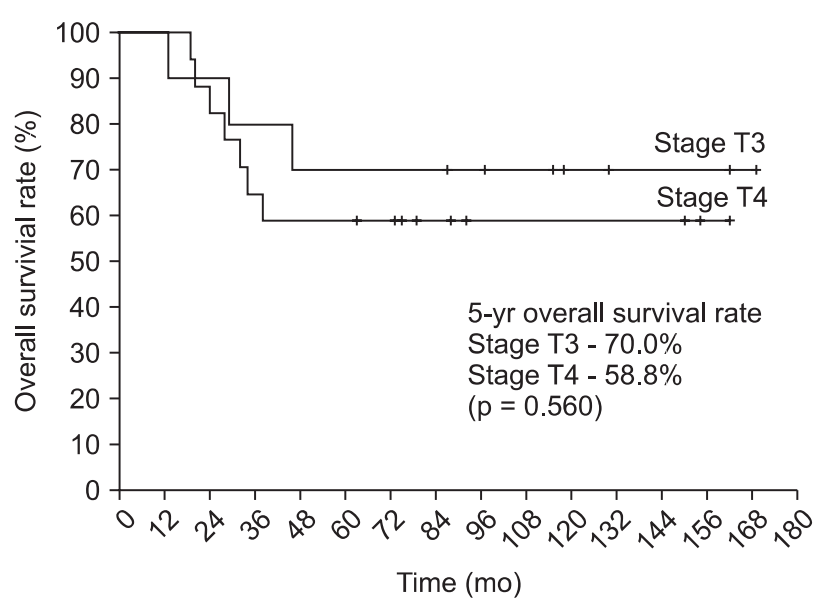

Fig. 2. Overall survival rates of each stage group.

with the stage T3 and T4 were 70.0\% and 58.8\%, respectively ( $p$ $=0.560$ ) (Fig. 2). There was no patient died from the treatment related acute and late complications.

\section{Normal tissue complications}

Table 4 presents the incidence of acute toxicities and late complication after combined neoadjuvant therapy. After IAC, overall 5 patients showed grade 3 acute toxicities. One patient had the moderate skin erythema. Two patients needed dose reduction due to moderate to severe leukocytopenia. All patients showed mild (CTC grade 1-2) mucositis, nausea, or fatigue. Three patients had grade 3 oral mucositis. Five patients reported grade 3 acute toxicities (i.e., nasal bleeding, mucositis, and abscess at the catheter drain site) after RT. There was no severe surgical complication reported. 
Table 4. Rates of late normal tissue complications

\begin{tabular}{llc}
\hline \multicolumn{1}{c}{ Site and type } & Grade 2 & $\geq$ Grade 3 \\
\hline $\begin{array}{l}\text { Orbital } \\
\text { Optic neuropathy }\end{array}$ & & $1(4)$ \\
Retinopathy & $1(4)$ & \\
Cataract & $3(11)$ & \\
$\quad$ Dry eye & $7(26)$ & $2(7)$ \\
Skin and mucosa & & \\
$\quad$ Xerostomia & $6(22)$ & $1(4)$ \\
Sinusitis & $9(33)$ & $2(7)$ \\
Telangiectasia & $2(7)$ & \\
Subcutaneous fibrosis & $5(19)$ & \\
Other & & \\
Nasolacrimal duct stenosis & $2(7)$ & $1(4)$ \\
Trismus & $2(7)$ & \\
Hearing loss & $1(4)$ & \\
\hline
\end{tabular}

Values are presented as number (\%).

Most patients had shown varying degrees of late skin and oral mucosa injury. Xerostomia and chronic sinusitis were relatively common. Severe subcutaneous fibrosis was rare in the patients who received the total radiation dose $\leq 50.4$ Gy. However, severe optic nerve and retinal injury were only reported in the patients received additional RT after the surgery. They received more than $60 \mathrm{~Gy}$ to the primary tumor area near the orbit. There was no statistical difference in the rates of complications between the stage $\mathrm{T} 3$ and $\mathrm{T} 4$ subgroup $(p=0.232)$. One patient with the tumor located laterally had experienced hearing loss in the ipsilateral ear. However it was unclear whether it was caused by the tumor itself or the treatment effect. There was no severe late complication related with the IAC.

\section{Re-treatment after local and/or regional recurrence}

The patients with the local and/or regional relapse were treated with either surgery alone or surgery with RT. Total 11 patients received re-treatment for local recurrence. Surgery alone was performed for six patients, and surgery and RT was done for five patients. Two patients received neck RT only. Three patients were re-treated with intensity modulated RT to the maxillary area. Relapse-free rate of the re-treatment for the first year was about $54 \%$.

\section{Discussion and Conclusion}

Our study demonstrated the long-term follow-up results of IAC and RT as a neoadjuvant modality for the treatment of locally advanced non-metastatic maxillary sinus cancer. Neoadjuvant
Table 5. Univariate analysis of prognostic factors

\begin{tabular}{lcc}
\hline \multicolumn{1}{c}{ Variable } & $\begin{array}{c}\text { Local } \\
\text { recurrence }\end{array}$ & $\begin{array}{c}\text { Overall } \\
\text { survival }\end{array}$ \\
\hline Age (<60 vs. $\geq 60)$ & 0.647 & 0.534 \\
Stage (T3 vs. T4) & 0.568 & 0.560 \\
Chemotherapy response ( $\geq$ PR vs. <PR) & 0.839 & 0.773 \\
Type of surgery (total maxillectomy or & 0.228 & 0.286 \\
craniofacial resection vs. < partial & & \\
$\quad$ maxillectomyor endoscopic resection) & & \\
RT dose ( $\geq 50.4$ Gy vs. <50.4 Gy) & 0.154 & 0.358 \\
Margin status (close or positive margin & 0.290 & 0.670 \\
$\quad$ vs. negative margin) & & \\
Tumor extent (orbit or skull base & 0.045 & 0.281 \\
involvement vs. not involved) & & \\
\hline
\end{tabular}

IAC and RT had shown the high rates of treatment response and tumor down-staging. Pathologic diagnosis confirmed the complete resection of the tumor in about $80 \%$ cases of the total patients. In addition we had found IAC or RT related complications were not significantly increased during the follow-up periods. Nevertheless, after a median follow-up of 5 years, the majority of the disease recurrences were local failure. Most common sites of local failure were periorbital region and base of skull. These areas were often received the lower radiation doses than the other areas due to avoiding the severe orbital complications (Table 5).

In many previously reported studies of maxillary sinus carcinoma, local recurrence remains the leading cause of treatment failure. Blanco et al. [4] reported long-term outcomes of radiotherapy in 106 patients with paranasal sinus carcinoma for more than three decades. Most patients treated with RT and/ or surgery without chemotherapy. The 5-year local control rate and overall survival rate were $58 \%$ and $27 \%$, respectively, even though aggressive local treatment was performed with combined surgery. They also noted that combined modality treatment slightly improved disease-free survival.

Neoadjuvant chemotherapy can increase the resectability of primary tumor when tumor involved or located close to critical organs. Advanced maxillary sinus cancer often involves orbit or base of skull. Extensive surgery or higher dose RT may cause severe defects or complications in these regions. Currently, data regarding the neoadjuvant concurrent chemoradiotherapy combined with surgery is limited. In addition, optimal sequence of chemotherapy and radiotherapy is unknown yet. Isobe et al. [5] showed improved local control and survival rate of 124 locally advanced maxillary sinus cancer patients treated with three different chemotherapy 
schedules (i.e., neoajuvant chemotherapy, concurrent chemoradiotherapy, and neoadjuvant chemotherapy plus concurrent chemoradiotherapy). In that study, 93\% of the patients had a T3 or T4 disease. No significant difference was found between the results of administration sequences of chemotherapeutic agents. Although other studies revealed the efficacy of the neoadjuvant systemic chemotherapy, the numbers of enrolled patients were not enough to determine apparently the impact on the efficacy.

Intra-arterial approach was ideally designed to deliver chemotherapeutic agents efficiently to the localized tumor area without further increasing the systemic toxicity of chemotherapy. Several IAC trials have been investigated in the treatment of maxillary sinus carcinoma for several decades. Various combined modality and schedules have been reported. In the current study, we have performed sequential RT after neoadjuvant IAC with 5-FU. However, concomitant RT delivery can maximize the therapeutic effect. Yoshimura et al. [6] also conducted the study of trimodal combination therapy with an intra-arterial 5-FU (daily 125 to $250 \mathrm{mg}$ ) infusion combined with concurrent RT. There were 29 local recurrences among total 110 patients. Their 5 -year cause-specific survival and overall survival rates were $71 \%$ and $63 \%$, respectively. They reported total sixty events of late complications in 42 patients. Thirty-three of the patients had eye complications. The majority of those eye complications were cataract.

Even though 5-FU is less toxic than cisplatin, intra-arterial cisplatin is often used to advance the chemotherapeutic effect. Samant et al. [7] reported the results of concomitant intra-arterial cisplatin (150 mg/m² per week) chemotherapy combined with preoperative RT. Preoperative RT was delivered in 5 weeks, total dose of $50 \mathrm{~Gy}$, with concomitant use of 3 to 4 weekly infusion of intra-arterial cisplatin followed by planned surgery. Actuarial overall survival rates at 2 and 5 years were $68 \%$ and $53 \%$, respectively. They have reported few treatmentlimiting toxic effects.

With advanced imaging techniques, it became possible to select direct feeding vessels around the primary tumor. Recently, Shiga et al. [8] had evaluated the efficacy of the superselective intra-arterial cisplatin infusion therapy. They treated the patients with either concurrent chemo-RT or surgery with postoperative RT. Both groups had a 2-cycle of the superselective IAC with cisplatin $\left(100 \mathrm{mg} / \mathrm{m}^{2}\right)$ before the treatments. From total 50 patients, 25 patients were treated with superseletive IAC with RT and the other 25 patients underwent surgery with postoperative RT. Patients group with surgery had shown better disease free survival rate than concurrent chemo-RT group (75\% vs. 62\%). From subgroup analysis, 5-year overall survival rate and local control rate were $87 \%$ and 100\%, respectively, in T4 stage patient treated with combined surgery group. They concluded that the surgery group had better survival outcomes than the RT group.

Due to the complexity of the anatomical structures in the vicinity of paranasal sinuses, complete resection of tumors in those areas is still challenging. The limited extent of surgery may have a significant impact on the treatment outcome. Resto et al. [9] have reported an analysis of outcomes related to the extent of surgery in the patients with locally advanced sinonasal malignancies. Three types of surgery-complete resection, partial resection, and biopsy were compared in the study which showed that high-dose RT resulted in excellent local control rates irrespective of the extent of surgery. However, complete resection had significantly improved disease-free survival rate and decreased the rate of distant metastasis.

With the recent advances in high precision RT techniques, the toxicities related to the RT have decreased and the ability of target coverage has increased. In the management of maxillary sinus cancers, the role of these modern RT techniques also has been investigated. Hoppe et al. [10] performed a retrospective analysis of patients with paranasal sinus cancer treated with modern RT techniques. They found that the overall survival and local control were comparable with other studies, but the rate of grade 3 or more ophthalmic toxicity was lower than those in the older studies using conventional RT techniques. Chen et al. [11] reported the similarly results in which the incidence of complications has significantly declined.

Our study has shown that neoadjuvant IAC and RT combined with surgery is relatively safe and effective regimen regardless of the long-term follow-up. Currently, most institutes widely use cisplatin when performing IAC for better tumor response, however, minimizing the toxicities is still challenging. We did not initially use the high precision RT technique in this study. With this new RT technique can decrease local failure in the region of the orbit and the base of skull. For further maximizing the therapeutic ratio, the use of better chemotherapeutic agents, super selective delivery methods, high precision RT technique, and minimal invasive surgery may improve the local control and survival outcomes.

In our study, small numbers of locally recurrent patients received the salvage re-irradiation with intensity-modulated $\mathrm{RT}$ after surgical resection. These treatments were relatively 
safe and the rate of severe toxicity was comparable to the initial treatment. Further studies with more patients and longer follow-up may confirm these results.

In conclusion, neoadjuvant IAC and RT in the patients with non-metastatic locally advanced or inoperable maxillary cancer was an effective and feasible treatment option for local tumor control with decreasing chemotherapy related systemic toxicity. Nevertheless, local failures were still the major causes of deaths. With intention to improve local tumor control and survival outcome, better RT regimen (i.e., higher dose delivery with precision RT techniques) and complete surgical management may be required. Further investigations are required to determine the optimal IAC, $R T$, and surgical treatment schedule and chemotherapy regimens.

\section{Conflict of Interest}

No potential conflict of interest relevant to this article was reported.

\section{Acknowledgments}

This work was supported by a 2-year research grant of Pusan National University.

\section{References}

1. Roush GC. Epidemiology of cancer of the nose and paranasal sinuses: current concepts. Head Neck Surg 1979;2:3-11.

2. Dulguerov $P_{1}$ Jacobsen MS, Allal AS, Lehmann $W$, Calcaterra T. Nasal and paranasal sinus carcinoma: are we making prog- ress? A series of 220 patients and a systematic review. Cancer 2001;92:3012-29.

3. Ganly I, Patel SG, Singh B, et al. Craniofacial resection for malignant paranasal sinus tumors: report of an International Collaborative Study. Head Neck 2005;27:575-84.

4. Blanco Al, Chao KS, Ozyigit $\mathrm{G}$, et al. Carcinoma of paranasal sinuses: long-term outcomes with radiotherapy. Int J Radiat Oncol Biol Phys 2004;59:51-8.

5. Isobe $K$, Uno T, Hanazawa $T$, et al. Preoperative chemotherapy and radiation therapy for squamous cell carcinoma of the maxillary sinus. Jpn J Clin Oncol 2005;35:633-8.

6. Yoshimura R, Shibuya $H_{1}$ Ogura I, et al. Trimodal combination therapy for maxillary sinus carcinoma. Int J Radiat Oncol Biol Phys 2002;53:656-63.

7. Samant $S$, Robbins KT, Vang M, Wan J, Robertson J. Intraarterial cisplatin and concomitant radiation therapy followed by surgery for advanced paranasal sinus cancer. Arch Otolaryngol Head Neck Surg 2004;130:948-55.

8. Shiga K, Yokoyama J, Hashimoto $S$, et al. Combined therapy after superselective arterial cisplatin infusion to treat maxillary squamous cell carcinoma. Otolaryngol Head Neck Surg 2007;136:1003-9.

9. Resto VA, Chan AW, Deschler DG, Lin DT. Extent of surgery in the management of locally advanced sinonasal malignancies. Head Neck 2008;30:222-9.

10. Hoppe BS, Stegman LD, Zelefsky MJ, et al. Treatment of nasal cavity and paranasal sinus cancer with modern radiotherapy techniques in the postoperative setting: the MSKCC experience. Int J Radiat Oncol Biol Phys 2007;67:691-702.

11. Chen AM, Daly ME, Bucci MK, et al. Carcinomas of the paranasal sinuses and nasal cavity treated with radiotherapy at a single institution over five decades: are we making improvement? Int J Radiat Oncol Biol Phys 2007;69:141-7. 